\title{
Particles and vacuum for perturbative and nonperturbative Einstein-Rosen gravity
}

\author{
J. Fernando Barbero G. \\ Instituto de Matemáticas y Física Fundamental, CSIC, Serrano 113bis, 28006 Madrid, Spain \\ Guillermo A. Mena Marugán \\ Instituto de Estructura de la Materia, CSIC, Serrano 121, 28006 Madrid, Spain \\ Eduardo J. S. Villaseñor \\ Departamento Matemáticas, Escuela Politécnica Superior, Universidad Carlos III de Madrid, \\ Avenida de la Universidad 30, 28911 Leganés, Spain \\ (Received 27 May 2004; published 20 August 2004)
}

\begin{abstract}
We discuss the connection between the Fock space introduced by Ashtekar and Pierri for Einstein-Rosen waves and its perturbative counterpart based on the concept of a particle that arises in linearized gravity with a de Donder gauge. We show that the gauge adopted by Ashtekar and Pierri is indeed a generalization of the de Donder gauge to full (i.e., non-linearized) cylindrical gravity. This fact allows us to relate the two descriptions of the Einstein-Rosen waves analyzed here (the perturbative one and that made by Ashtekar and Pierri) by means of a simple field redefinition. Employing this redefinition, we find the highly non-linear relation that exists between the annihilation and creation-like variables of the two approaches. We next represent the particle-like variables of the perturbative approach as regularized operators, introducing a cutoff. These can be expanded in powers of the annihilation and creation operators of the Ashtekar-Pierri quantization, each additional power being multiplied by an extra square root of ( $\hbar$ times) the three-dimensional gravitational constant, $\sqrt{G}$. In principle, the perturbative vacuum may be reached as the limit of a state annihilated by these regularized operators when the cutoff is removed. This state can be written as the vacuum of the Ashtekar-Pierri quantization corrected by a perturbative series in $\sqrt{G}$ with no contributions from particles with energies above the cutoff. We show that the first-order correction is in fact a state of infinite norm. This result is interpreted as indicating that the Fock quantizations in the two approaches are unitarily inequivalent and, in any case, proves that the perturbative vacuum is not analytic in the interaction constant. Therefore a standard perturbative quantum analysis fails.

DOI: 10.1103/PhysRevD.70.044028

PACS number(s): 04.60.Ds, 04.60.Kz, 04.62.+v, 11.15.Bt
\end{abstract}

\section{INTRODUCTION}

Solutions to general relativity in vacuo with whole cylindrical symmetry seem to have been first found by Beck [1] and then rediscovered by Einstein and Rosen (ER) in the search for spacetimes that could describe the propagation of gravitational waves [2]. By whole cylindrical symmetry [3] we understand the existence (in topologically trivial spacetimes) of two linearly independent, commuting, and hypersurface orthogonal Killing vector fields, one of them rotational and the other translational. Among the motivations for the study of these solutions was Einstein's belief that one of the fundamental problems of physics (at least at his time) was the lack of a satisfactory theory of radiation, especially in the presence of the gravitational field [4].

The ER solutions are cylindrical gravitational waves with linear polarization. Cylindrical waves with general polarization, whose Killing vector fields are not hypersurface orthogonal, were originally analyzed by Jordan, Ehlers and Kundt and by Kompaneets [5].

The ability to provide a model with the field complexity of general relativity, but with known exact solutions which describe gravitational waves, has endowed the family of ER spacetimes with a prominent role in the analysis of the quantization of gravitational systems [6-16]. Kuchař pioneered this line of work by discussing the canonical quantization of these cylindrical waves [6]. A key remark in this discussion is that the dynamics of the ER spacetimes is equivalent to that of a cylindrically symmetric, massless scalar field propagating on an auxiliary Minkowski background. Thanks to this fact, one can recast the system as three-dimensional gravity coupled to a scalar field with rotational symmetry. This was precisely the approach followed by Allen to further explore the quantization of the model, studying regularization issues and the relevance of the quantum fluctuations around the vacuum [7].

Employing this three-dimensional formulation, a consistent and essentially complete quantization of the ER waves was obtained some years ago by Ashtekar and Pierri (AP) [8]. This quantization was achieved after a careful treatment of the regularity conditions at the symmetry axis, on the one hand, and of the boundary conditions at spatial infinity that ensure asymptotic flatness in cylindrical gravity $[8,17]$, on the other. The quantization accounts as well for certain functional analytic subtleties that arise in the regularization of metric operators. Actually, some of these subtleties were later revisited by Varadarajan [9]. The definition and regularization of the metric operators, not from the perspective of three-dimensional gravity coupled to a scalar field, but from a purely gravitational, four-dimensional perspective was discussed in Ref. [10].

This quantum framework has allowed to show that there 
exist unexpectedly large quantum effects in the model, at least in the asymptotic region [11-13] and the cylindrical axis [10]. A more detailed study of the consequences of the vacuum fluctuations for microcausality, including the smearing of light cones all over the spacetime and the blurring of the symmetry axis, has recently been carried out in Ref. [14].

In the quantization proposed by Ashtekar and Pierri, the Hilbert space is the Fock space corresponding to the rotationally symmetric scalar field that propagates in the threedimensional, auxiliary Minkowski spacetime. There exist two relevant notions of evolution in this Hilbert space: one associated with the auxiliary Minkowski time and another with the physical time $[8,14]$. In the former case, the dynamics is dictated by the Hamiltonian of the axisymmetric, massless scalar field, $H_{0}$, while in the latter the Hamiltonian is a non-linear, bounded function of it, $H=\left(1-e^{-4 G_{3} H_{0}}\right) /\left(4 G_{3}\right)[18-20]$. Here, $G_{3}$ is the threedimensional gravitational constant or, equivalently, the effective Newton constant per unit length in the direction of the axis [10].

The difference between the dynamical generators arises because the presence of energy in the gravitational waves causes a deficit angle at spatial infinity that affects the norm of the asymptotic, time-like Killing vector. Since this norm must be unity for the physical time, one must consider an energy dependent change of time that leads to the above transformation in the Hamiltonian. In fact, the emergence of a bounded physical Hamiltonian proportional to the deficit angle produced by the wave is a feature not just of ER gravity, but of cylindrical gravitational waves with general polarization (even in the presence of spinning strings) [21,22].

Regardless of which Hamiltonian is considered to govern the quantum dynamics, $H_{0}$ or $H$, the Fock spaces and quantizations obtained in both cases are equivalent, in as much as the corresponding evolution operators are unitary and the two initial time copies are exactly the same [14]. However, a question that has not been addressed yet in the literature is whether the Fock space employed in the AP quantization is the kind of Fock space that one would introduce in a perturbative treatment of the ER model and, if they differ, what relation exists between them. The main aim of this article is to discuss this issue. This is a fundamental question in order to answer whether one can attain or not the correct nonperturbative results by adopting a perturbative approach.

In a perturbative formalism, one would adopt as metric variables linear combinations of the difference between the Minkowski background and the actual spacetime metric, expanding the gravitational action in powers of them. The quadratic term provides the action of linearized gravity, while the higher-order terms can be regarded as describing interactions. At this stage, it is convenient to adopt a gauge that simplifies the linearized equations. A frequently used gauge is the de Donder or Lorentz gauge [23], in which the linearized gravitational equations reduce to wave equations, so that one easily arrives at a notion of particle.

We will see that the gauge fixing introduced by Ashtekar and Pierri is nothing but a generalization of the de Donder gauge from linearized to full ER gravity. Therefore, adopting it as a valid gauge (with a clear interpretation in linearized gravity), the relation between the AP and the perturbative treatments will straightforwardly follow from the transformation on the configuration space that connects the metric variables used in each of the two descriptions. In particular, this transformation, when completed into a canonical one, will provide the relation between the particle-like variables of the two formalisms.

The plan of the work is as follows. We first review the ER model and the most important aspects of the AP quantization in Sec. II. In Sec. III we introduce a description of the ER waves in terms of fields that are linear in the excess of the metric around the Minkowski background and translate to them the AP gauge fixing. In Sec. IV we adapt to this description the discussion of Ref. [14] about the linearization of the model. Section $\mathrm{V}$ proves that the linearization of the AP gauge is a de Donder gauge. Furthermore, while in general relativity the de Donder gauge leaves some ambiguity in the choice of coordinates [23], the gauge is completely determined in linearized ER gravity when one imposes suitable regularity conditions, corresponding to a fixed location of the symmetry axis. Employing the transformation that maps the basic metric field of the reduced ER model in our description (linear in the excess around Minkowski) to the axisymmetric scalar field of the AP approach, we find in Sec. VI the relation between the creation and annihilation variables associated with each of the two fields considered. These variables are promoted to regularized operators in Sec. VII. Using them, we try to determine the perturbative vacuum in Sec. VIII. In particular, we investigate whether this vacuum can be reached from the non-perturbative one by means of a series expansion in (the square root of) the gravitational constant. The answer turns out to be in the negative, because the first correction to the non-perturbative vacuum would have an infinite norm, even if ultraviolet divergences are regulated with a cutoff. Finally, Sec. IX contains the conclusions.

In the rest of the paper, we call $G=G_{3} \hbar$, and adopt a system of units in which $\hbar=c=1$, with $\hbar$ being Planck constant and $c$ the speed of light. Note that $G_{3}$ is an inverse energy, whereas $G$ has dimensions of length.

\section{ASHTEKAR-PIERRI QUANTIZATION}

The ER waves are linearly polarized, cylindrical waves in vacuum general relativity. They can be described by the metric $[8,10]$

$$
\begin{aligned}
d s^{2}= & e^{-\psi}\left[-N^{2} d t^{2}+e^{\gamma}\left(d R+N^{R} d t\right)^{2}+(8 G r)^{2} d \theta^{2}\right] \\
& +e^{\psi} d Z^{2}
\end{aligned}
$$

Here, $Z \in \mathbb{R}$ is the coordinate of the symmetry axis, $\theta \in S^{1}$ corresponds to the axial coordinate, $R \in \mathbb{R}^{+}$will be called the radial coordinate, $N^{R}$ describes the radial component of the shift vector and $N$ is the lapse function. Owing to the cylindrical symmetry, all metric functions $N^{R}, N, \psi, r$, and $\gamma$ depend only on the time $t$ and on the radial coordinate $R$. We follow the convention that the dimensionality of the spacetime interval is carried by the coordinates $t, R$, and $Z$, while the metric fields are dimensionless [24]. 
The gravitational action, endowed with surface terms suitable for a fixed metric on the boundaries (namely, the initial and final $t$ sections and an exterior cylinder $R=R_{f}$ in the limit $R_{f} \rightarrow \infty$ [10]), takes the form [6,17]

$$
S=\int_{t_{1}}^{t_{2}} d t\left[-\mathcal{H}+\int_{0}^{\infty} d R\left(p_{\gamma} \dot{\gamma}+p_{r} \dot{r}+p_{\psi} \dot{\psi}\right)\right],
$$

where the $p$ 's are the momenta canonically conjugate to the metric fields, the overdot stands for the derivative with respect to $t$, and $\mathcal{H}$ is the total Hamiltonian:

$$
\mathcal{H}=\frac{1}{4 G_{3}}\left(1-e^{-\gamma_{\infty} / 2}\right)+\int_{0}^{\infty} d R\left[N C+N^{R} C^{R}\right] .
$$

The first contribution is a boundary term at infinity, with $\gamma_{\infty}:=\gamma(R \rightarrow \infty)$. Besides, the Hamiltonian constraint $C$ and the (radial) diffeomorphism constraint $C^{R}$ are

$$
\begin{aligned}
C & =e^{-\gamma / 2}\left[2 r^{\prime \prime}-\gamma^{\prime} r^{\prime}-p_{\gamma} p_{r}+\frac{p_{\psi}^{2}}{2 r}+\frac{r\left(\psi^{\prime}\right)^{2}}{2}\right], \\
C^{R} & =-2 p_{\gamma}^{\prime}+p_{\gamma} \gamma^{\prime}+p_{r} r^{\prime}+p_{\psi} \psi^{\prime} .
\end{aligned}
$$

The prime denotes the derivative with respect to $R$.

The Lagrangian of the model can be straightforwardly obtained by means of a Legendre transformation using the relation between momenta and time derivatives of the metric that the Hamilton equations provide

$$
\begin{aligned}
& p_{\gamma} N e^{-\gamma / 2}=-\dot{r}+N^{R} r^{\prime}, \\
& p_{r} N e^{-\gamma / 2}=-\dot{\gamma}+2\left(N^{R}\right)^{\prime}-N^{R} \gamma^{\prime}, \\
& p_{\psi} N e^{-\gamma / 2}=r \dot{\psi}-N^{R} r \psi^{\prime} .
\end{aligned}
$$

All metric fields, as well as their momenta, are subject to boundary conditions that ensure the regularity at the symmetry axis, the asymptotic flatness at spatial infinity (with a possible deficit angle), and a well-posed Hamiltonian dynamics. We refer to the work of Ashtekar and Pierri [8] for details about these conditions. In particular, we assume that all fields are $C^{\infty}$ everywhere, that at the axis

$$
\begin{aligned}
& \gamma(R=0)=0, \quad N^{R}(R=0)=0, \\
& \rho(R=0)=0, \quad \rho^{\prime}(R=0)=0,
\end{aligned}
$$

where we have called $\rho:=r-R /(8 G)$, and that at spatial infinity,

$$
\psi=O\left(\frac{1}{R}\right), \quad \rho=O(1) .
$$

We say that a function $f$ is of asymptotic order $O\left(R^{-a}\right)$ if the products $R^{a} f, R^{a+1} f^{\prime}$, and $R^{a+2} f^{\prime \prime}$ admit limits as $R$ tends to infinity [8]. In addition, we note that, for the stability under diffeomorphisms of the regularity condition that $\gamma$ vanish at the axis, one must further restrict the shift vector to satisfy [25]

$$
\left.\left\{\gamma(\widetilde{R}), \int d R N^{R} C^{R}\right\}\right|_{\widetilde{R}=0}=\left.2\left(N^{R}\right)^{\prime}\right|_{\widetilde{R}=0}=0,
$$

where the curved brackets denote Poisson brackets.

As shown by Ashtekar and Pierri [8], the gauge freedom corresponding to the Hamiltonian and radial constraints can be totally removed by imposing, respectively, the conditions

$$
\chi^{R}:=r-\frac{R}{8 G}=0, \quad \chi:=p_{\gamma}=0 .
$$

In this way, one arrives at a reduced model whose only degree of freedom is the scalar field $\psi$. The reduced metric is given by Eq. (2.1) with $N^{R}=0,8 G r=R$ and

$$
\gamma=\int_{0}^{R} d \widetilde{R} \frac{\widetilde{R}}{2}\left[\left(\psi^{\prime}\right)^{2}+\frac{\left(8 G p_{\psi}\right)^{2}}{\widetilde{R}^{2}}\right] .
$$

The reduced dynamics in the time $t$ is generated by the physical Hamiltonian $H=\left(1-e^{-4 G_{3} H_{0}}\right) /\left(4 G_{3}\right)$, where $H_{0}:=\gamma_{\infty} /\left(8 G_{3}\right)$ is (up to a constant factor) the Hamiltonian that would correspond to a free, axisymmetric, massless scalar field $\psi$ in three dimensions.

The evolution of the field $\psi$ gets considerably simplified if one introduces the energy dependent change of time $T=e^{-4 G_{3} H_{0}} t$. In this auxiliary time, the dynamics is dictated precisely by the free-field Hamiltonian $H_{0}$, so the equation of motion for $\psi$ is just a wave equation with rotational symmetry in a three-dimensional Minkowski spacetime with coordinates $(T, R, \theta)$. The classical solutions with regularity at the axis have the form

$$
\psi(R, T)=\sqrt{4 G} \int_{0}^{\infty} d k J_{0}(R k)\left[A(k) e^{-i k T}+A^{\dagger}(k) e^{i k T}\right],
$$

where $J_{0}$ is the zeroth-order Bessel function [26]. The function $A(k)$ and its complex conjugate $A^{\dagger}(k)$ are fixed by the initial conditions and play the role of annihilation and creation variables. In terms of them, the free-field Hamiltonian can be written as $H_{0}=\int_{0}^{\infty} d k k A^{\dagger}(k) A(k)$.

The quantization of the reduced ER model can be carried out following standard techniques. We introduce a Fock space in which $\hat{\psi}(R, 0)$, the quantum counterpart of $\psi(R, 0)$, is an operator-valued distribution [27]. Its action is determined by those of $\hat{A}(k)$ and $\hat{A}^{\dagger}(k)$, annihilation and creation operators with non-vanishing commutators:

$$
\left[\hat{A}(k), \hat{A}^{\dagger}(\widetilde{k})\right]=\delta(k, \widetilde{k}) .
$$

Explicitly,

$$
\hat{\psi}(R, 0)=\sqrt{4 G} \int_{0}^{\infty} d k J_{0}(R k)\left[\hat{A}(k)+\hat{A}^{\dagger}(k)\right]
$$

and the Fock space is constructed over the Hilbert space of square integrable functions on the positive real axis, $L^{2}\left(\mathbb{R}^{+}, d k\right)$. In this space, the free-field Hamiltonian is represented by the self-adjoint operator 


$$
\hat{H}_{0}=\int_{0}^{\infty} d k k \hat{A}^{\dagger}(k) \hat{A}(k) .
$$

Via the spectral theorem, we can then promote the physical Hamiltonian to a bounded operator $\hat{H}$. The evolution in the time parameters $T$ and $t$ are thus respectively given by the unitary operators $\hat{U}_{0}(T)=e^{-i T \hat{H}_{0}}$ and $\hat{U}(t)=e^{-i t \hat{H}}$.

In order to arrive at a well-defined operator for $\psi$, it is necessary to regularize the quantum field (2.10) [10]. This can be done by smearing the Bessel function $J_{0}(R k)$ with a square integrable function $g \in L^{2}\left(\mathbb{R}^{+}, d k\right)$ :

$$
\hat{\psi}(R \mid g)=\sqrt{4 G} \int_{0}^{\infty} d k g(k) J_{0}(R k)\left[\hat{A}(k)+\hat{A}^{\dagger}(k)\right] .
$$

It is then possible to define meaningful metric operators, e.g. for the diagonal $Z$ and $\theta$ components (at the initial time), by exponentiating the regulated version of the field, $e^{ \pm \hat{\psi}(R \mid g)}$ [10]. For simplicity, we will restrict in the following to regulators that correspond to a cutoff $k_{c}$, so that

$$
g(k)= \begin{cases}1 & \text { if } k \leqslant k_{c}, \\ 0 & \text { if } k>k_{c} .\end{cases}
$$

In particular, we have $g^{2}(k)=g(k)$.

\section{NEW METRIC FIELDS}

We will introduce now a different field parametrization for the Einstein-Rosen metric such that the new fields are linear in the metric excess around Minkowski spacetime:

$$
\begin{aligned}
d s^{2}= & -(1-2 \bar{N}-\bar{\psi}) d t^{2}+2 \bar{N}^{R} d t d R+(1+\bar{\gamma}-\bar{\psi}) d R^{2} \\
& +\left(R^{2}-R^{2} \bar{\psi}+16 G R \bar{\rho}\right) d \theta^{2}+(1+\bar{\psi}) d Z^{2} .
\end{aligned}
$$

Obviously, when all the fields $\left(\bar{N}, \bar{N}^{R}, \bar{\psi}, \bar{\rho}, \bar{\gamma}\right)$ vanish, we recover the Minkowski solution. Moreover, our new parametrization is specially suited for the study of linearized gravity, since it reproduces the linearization of the spacetime metric (2.1) around the Minkowski background [14] [apart from the notation with over-bars and with $\rho=r-R /(8 G)]$. Hence, at first order one can interpret our new metric fields as the linearization of those used in the AP formulation.

The exact relation between both sets of fields is found by identifying the metric expressions. One gets

$$
\begin{aligned}
\psi & =\ln (1+\bar{\psi}), \\
\gamma & =\ln [(1+\bar{\psi})(1+\bar{\gamma}-\bar{\psi})], \\
8 G r & =\sqrt{1+\bar{\psi}} \sqrt{R^{2}-R^{2} \bar{\psi}+16 G R \bar{\rho}}, \\
N & =\sqrt{1+\bar{\psi}} \sqrt{1+2 \bar{N}-\bar{\psi}-\frac{\left(\bar{N}^{R}\right)^{2}}{1+\bar{\gamma}-\bar{\psi}}},
\end{aligned}
$$

$$
N^{R}=\frac{\bar{N}^{R}}{1+\bar{\gamma}-\bar{\psi}} .
$$

In principle, if one insists on imposing that the fields on the left hand side be real, the range of the new metric fields should be properly restricted. Note also that the transformation is always well defined (even with these reality conditions) in a neighborhood of the Minkowski solution, i.e., for small over-barred fields and hence for the linearized theory.

The above transformation can be easily completed into a canonical one. Old and new momenta are related by

$$
\begin{aligned}
& p_{\psi}=(1+\bar{\psi}) p_{\bar{\psi}}+(2 \bar{\psi}-\bar{\gamma}) p_{\bar{\gamma}}+\left(\frac{R}{8 G} \bar{\psi}-\bar{\rho}\right) p_{\bar{\rho}}, \\
& p_{\gamma}=(1+\bar{\gamma}-\bar{\psi}) p_{\bar{\gamma}}, \\
& p_{r}=\sqrt{1-\bar{\psi}+\frac{16 G \bar{\rho}}{R} \frac{p_{\bar{\rho}}}{\sqrt{1+\bar{\psi}}} .}
\end{aligned}
$$

Using these expressions, it is not difficult to write the constraints of the ER model in terms of the new canonical variables. The associated gauge freedom can be eliminated by imposing conditions equivalent to those employed by Ashtekar and Pierri, namely

$$
\bar{\chi}^{R}:=(1+\bar{\psi}) \bar{\rho}-\frac{R}{16 G} \bar{\psi}^{2}=0, \quad \bar{\chi}:=p_{\bar{\gamma}}=0 .
$$

Taking into account relations (3.2), (3.3), and (3.4), one can check that these conditions are identical to the gauge fixing requirements (2.7) except for metric-dependent, global multiplicative factors that differ from zero for almost all values of the metric fields and, in particular, in a neighborhood of their origin (the Minkowski background).

The reduction of the model can be carried out by the same procedure followed by Ashtekar and Pierri, translated to the new variables, so we will not repeat the details here. Perhaps the only noticeable point is that, owing to the dependence of the gauge fixing condition $\bar{\chi}^{R}$ on $\bar{\psi}$, the momentum canonically conjugate to $\bar{\psi}$ after reduction, $\bar{P}_{\bar{\psi}}$, does not coincide with the original one $p_{\bar{\psi}}$ (in other words, the Poisson and Dirac brackets of $\bar{\psi}$ and $p_{\bar{\psi}}$ differ). One finds instead

$$
\bar{P}_{\bar{\psi}}=\frac{2(1+\bar{\psi})}{2(1+\bar{\psi})+R \bar{\psi} \bar{\psi}^{\prime}(2+\bar{\psi})} p_{\bar{\psi}} .
$$

The only physical degree of freedom is described by $\bar{\psi}$. The reduced metric, regular at the axis and with asymptotically unit lapse, takes the form

$$
\begin{aligned}
d s^{2}= & (1+\bar{\gamma}-\bar{\psi})\left[-\frac{d t^{2}}{1+\bar{\gamma}_{\infty}}+d R^{2}\right]+\frac{R^{2}}{1+\bar{\psi}} d \theta^{2} \\
& +(1+\bar{\psi}) d Z^{2}
\end{aligned}
$$


with $\bar{\gamma}$ given by

$$
\begin{aligned}
(1+\bar{\gamma}-\bar{\psi})(1+\bar{\psi})= & \exp \left\{\int _ { 0 } ^ { R } d \widetilde { R } \frac { \widetilde { R } } { 2 } \left[\left(\frac{\bar{\psi}^{\prime}}{1+\bar{\psi}}\right)^{2}\right.\right. \\
& \left.\left.+\left(\frac{8 G \bar{P}_{\bar{\psi}}}{\widetilde{R}}\right)^{2}(1+\bar{\psi})^{2}\right]\right\} .
\end{aligned}
$$

In the reduced metric, $\bar{\gamma}_{\infty}$ denotes the limit when $R \rightarrow \infty$ of $\bar{\gamma}$. Recalling conditions (2.5) and relations (3.3), which in particular imply that $\bar{\psi}_{\infty}=0$, one easily checks that

$$
\begin{aligned}
1+\bar{\gamma}_{\infty}= & e^{\gamma_{\infty}}=\exp \left\{\int _ { 0 } ^ { \infty } d R \frac { R } { 2 } \left[\left(\frac{\bar{\psi}^{\prime}}{1+\bar{\psi}}\right)^{2}\right.\right. \\
& \left.\left.+\left(\frac{8 G \bar{P}_{\bar{\psi}}}{R}\right)^{2}(1+\bar{\psi})^{2}\right]\right\} .
\end{aligned}
$$

Finally, the action of the reduced model is

$$
S_{r}=\int_{t_{1}}^{t_{2}} d t\left[-\frac{1}{4 G_{3}}\left(1-\frac{1}{\sqrt{1+\bar{\gamma}_{\infty}}}\right)+\int_{0}^{\infty} d R \bar{P}_{\bar{\psi}} \dot{\bar{\psi}}\right] .
$$

The first contribution is therefore minus the reduced Hamiltonian. In fact, as one could expect, the above expressions for the reduced metric and action reproduce exactly those obtained in the AP treatment of the ER model, provided that the basic fields $\psi$ and $\bar{\psi}$ are related by $\psi=\ln (1+\bar{\psi})$ [see Eq. (3.2)] and their canonical momenta by

$$
p_{\psi}=\bar{P}_{\bar{\psi}}(1+\bar{\psi}) .
$$

This is precisely the change of momentum needed to complete our field redefinition into a canonical transformation for the reduced system. In other words, instead of changing the metric fields for the ER waves, completing the transformation into a canonical one, and reducing the system, one can simply proceed to redefine the field $\psi$ in the reduced model while preserving its canonical symplectic structure.

It is worth noting that, if one insists that the induced metric of the reduced model be positive, one should demand that the field $\psi$ be real or, equivalently, that $\bar{\psi}>-1$. In a perturbative analysis, however, the field $\bar{\psi}$ is directly assumed to be small and so the above reality condition would be obviated in practice.

\section{EINSTEIN-ROSEN WAVES IN LINEARIZED GRAVITY}

Let us consider the linearization of the ER model around the Minkowski solution. Since our new parametrization of the metric is linear in the excess around the flat background, the action of the linearized theory $\bar{S}_{l}$ can be obtained from that in general relativity by keeping only up to quadratic terms in our fields. Our discussion will essentially follow the lines presented in Ref. [14]. One starts with the Lagrangian form of the action (2.2), which can be easily deduced em- ploying the Hamilton equations (2.3). Next, one expresses it in terms of our new metric fields employing the transformations (3.2) and (3.3), and expands the result in powers of those fields. As we have commented, the action $\bar{S}_{l}$ is given by the terms quadratic in the fields. In deducing this action, one can get rid of several boundary terms by using the regularity and asymptotic conditions (2.4) and (2.5). Together with our field redefinitions, the latter of these sets of conditions implies that, at spatial infinity, the limits of $\bar{\psi}, R \bar{\psi}^{\prime}$, $\bar{\rho} / R$, and $\bar{\rho}^{\prime}$ vanish. The regularity conditions (2.4) guarantee in turn that [for generic values of $\bar{\psi}(R=0)] \bar{N}^{R}$ and $\bar{\rho}$ vanish at the axis and

$$
\bar{\gamma}(R=0)=16 G \bar{\rho}^{\prime}(R=0)=\frac{\bar{\psi}^{2}(R=0)}{1+\bar{\psi}(R=0)} .
$$

We notice also that the strict linearization of these relations between field values leads to $\bar{\gamma}(R=0)=\bar{\rho}^{\prime}(R=0)=0$.

Writing back the action in Hamiltonian form by means of a Legendre transformation, one gets [14]

$$
\begin{aligned}
& \bar{S}_{l}=\int_{t_{1}}^{t_{2}} d t\left[-\overline{\mathcal{H}}_{l}+\int_{0}^{\infty} d R\left(P_{\bar{\gamma}} \dot{\bar{\gamma}}+P_{\bar{\rho}} \dot{\bar{\rho}}+P_{\bar{\psi}} \dot{\bar{\psi}}\right)\right], \\
& \overline{\mathcal{H}}_{l}=\int_{0}^{\infty} d R\left[\frac{4 G P_{\bar{\psi}}^{2}}{R}+\frac{R\left(\bar{\psi}^{\prime}\right)^{2}}{16 G}-P_{\bar{\gamma}} P_{\bar{\rho}}+\bar{N} \bar{C}_{l}+\bar{N}^{R} \bar{C}_{l}^{R}\right] .
\end{aligned}
$$

Here, the $P$ 's denote the canonical momenta in the linearized model and, in terms of time derivatives of the metric, take the expressions

$$
P_{\bar{\gamma}}=\frac{\bar{N}^{R}}{8 G}-\dot{\bar{\rho}}, \quad P_{\bar{\rho}}=2\left(\bar{N}^{R}\right)^{\prime}-\dot{\bar{\gamma}}, \quad P_{\bar{\psi}}=\frac{R}{8 G} \dot{\bar{\psi}} .
$$

In addition, the linearized Hamiltonian and radial constraints are

$$
\bar{C}_{l}=2 \bar{\rho}^{\prime \prime}-\frac{\bar{\gamma}^{\prime}}{8 G}, \quad \bar{C}_{l}^{R}=\frac{P_{\bar{\rho}}^{-}}{8 G}-2 P_{\bar{\gamma}}^{\prime} .
$$

It has recently been shown [14] that the gauge freedom of the linearized system can be completely removed by demanding that

$$
\bar{\chi}_{l}^{R}:=\bar{\rho}=0, \quad \bar{\chi}_{l}:=P \bar{\gamma}_{\gamma}=0 .
$$

We point out that these conditions are just the linearization of the gauge fixing requirements (3.5) imposed on the full ER model or, equivalently, of the gauge fixing (2.7) introduced by Ashtekar and Pierri. The subsequent reduction can be carried out exactly as explained in Ref. [14]. The degrees of freedom of the system are $\bar{\psi}$ and its momentum. The reduced action is 


$$
\bar{S}_{2}=\int_{t_{1}}^{t_{2}} d t\left[-H_{0}+\int_{0}^{\infty} d R P_{\bar{\psi}} \dot{\bar{\psi}}\right] .
$$

Here, $H_{0}$ is again the Hamiltonian of a massless scalar field with rotational symmetry in three dimensions, and can be identified with $\gamma_{\infty} /\left(8 G_{3}\right)$ if, in Eq. (2.8), one evaluates $\gamma$ at the canonical pair $\left(\bar{\psi}, P_{\bar{\psi}}\right)$. At quadratic order in these fields, we see from Eq. (3.7) that $H_{0}$ coincides as well with $\bar{\gamma}_{\infty} /\left(8 G_{3}\right)$. Thus, the free-field Hamiltonian provides the reduced Hamiltonian of the model in linearized gravity.

Moreover, in the selected gauge, the three-dimensional metric of the linearized model is just Minkowski. The scalar field $\bar{\psi}$ determines the norm of the Killing vector $\partial_{Z}$, and appears in the reduced four-dimensional metric of the linearized theory in the form

$$
d s_{l}^{2}=(1-\bar{\psi})\left(-d T^{2}+d R^{2}+R^{2} d \theta^{2}\right)+(1+\bar{\psi}) d Z^{2} .
$$

We have renamed $T$ the time coordinate of the linearized system to emphasize that it can be identified with the auxiliary time of the reduced ER model analyzed in Sec. II, inasmuch as they are both Minkowskian in three dimensions and the corresponding evolution is generated by the free-field Hamiltonian $H_{0}$ in both cases.

\section{V. de DONDER GAUGE}

We want to prove now that the gauge chosen by Ashtekar and Pierri is a higher-order generalization of the de Donder gauge for linearized gravity, i.e. that the linearization of the AP gauge (which is precisely that imposed in Sec. IV to reduce the linearized system) is of de Donder type. Let us start with some notation. We call $h_{\mu \nu}=g_{\mu \nu}-\eta_{\mu \nu}$ the difference between the actual spacetime metric $g_{\mu \nu}$ and the flat metric $\eta_{\mu \nu}$ of the Minkowski background $\left[\eta_{\mu \nu}\right.$ $=(-1,1,1,1)$ in a Cartesian coordinate system $]$. Standard perturbative approaches to gravity analyze the gravitational interaction by means of an expansion in

$$
\bar{h}_{\mu \nu}=h_{\mu \nu}-\frac{h}{2} \eta_{\mu \nu}
$$

where $h=h_{\mu \nu} \eta^{\mu \nu}$ is the trace of $h_{\mu \nu}$. In a first-order approximation (namely, in linearized gravity), only terms up to quadratic in $\bar{h}_{\mu \nu}$ are maintained in the action. To go beyond that approximation, higher-order terms are treated perturbatively as interactions.

In the linearized theory, a frequently followed approach is to (partially) remove the gauge freedom by introducing a set of conditions that simplify the equations of motion for $\bar{h}_{\mu \nu}$, transforming them into wave equations [23], namely

$$
\bar{h}_{\mu \nu, \delta} \eta^{\nu \delta}=\bar{h}_{\mu \nu,}{ }^{\nu}=0
$$

These conditions are known as de Donder (or Lorentz) gauge, and provide an acceptable gauge fixing in linearized gravity, although they still leave some freedom in the choice of coordinates [23].

The most straightforward way to elucidate whether the gauge imposed in the AP analysis of the ER waves is a higher-order generalization of a de Donder gauge is to calculate the value of $\bar{h}_{\mu \nu}{ }^{\nu}$ in the reduced model of Sec. III. If $\bar{h}_{\mu \nu}{ }^{\nu}$ vanishes at first order in the fields, then the linearization of our gauge fixing conditions is indeed a de Donder gauge.

Changing from cylindrical coordinates $(R, \theta)$ to Cartesian ones $(x, y)$ and employing that, for any cylindrically symmetric field $f$,

$$
\partial_{x} f=\frac{x}{R} f^{\prime}, \quad \partial_{y} f=\frac{y}{R} f^{\prime}, \quad \partial_{Z} f=0
$$

a direct calculation shows that $\bar{h}_{Z \nu}{ }^{\nu}=0$ and

$$
\begin{aligned}
\bar{h}_{t \nu,}{ }^{\nu}= & -\frac{1}{2}\left[\frac{\bar{\gamma}_{\infty}}{1+\bar{\gamma}_{\infty}}(\dot{\bar{\gamma}}-\dot{\bar{\psi}})+\frac{\dot{\bar{\psi}} \bar{\psi}(2+\bar{\psi})}{(1+\bar{\psi})^{2}}\right], \\
\bar{h}_{x \nu,}{ }^{\nu}= & \frac{x}{2 R}\left[\frac{\bar{\gamma}_{\infty}}{1+\bar{\gamma}_{\infty}}\left(\bar{\gamma}^{\prime}-\bar{\psi}^{\prime}\right)-\frac{\bar{\psi}^{\prime} \bar{\psi}(2+\bar{\psi})}{(1+\bar{\psi})^{2}}\right] \\
+ & \frac{x}{R^{2}}\left(\bar{\gamma}-\frac{\bar{\psi}^{2}}{1+\bar{\psi}}\right),
\end{aligned}
$$

with a similar expression for $\bar{h}_{y \nu}{ }^{\nu}$ replacing $x$ with $y$ in the last equation. Here, $\bar{\gamma}$ is determined in terms of $\bar{\psi}$ and its momentum by Eq. (3.6). In particular, this value of $\bar{\gamma}$ is at least quadratic in the fields of the reduced model.

It is easy to check that, while the metric derivatives $\bar{h}_{\mu \nu}{ }^{\nu}$ do not generally vanish according to Eq. (5.1), their value is in fact equal to zero at linear order after the reduction of the system. As a consequence, the gauge that we have chosen for the analysis of the ER waves in linearized gravity is a de Donder gauge, and the gauge fixing selected by Ashtekar and Pierri is a valid generalization of it to the full (i.e., nonlinearized) model. In fact, a straightforward computation using the reduced metric (4.5) of the linearized model leads to the conclusion that $\bar{h}_{\mu \nu}=2 \bar{\psi} \delta_{\mu}^{Z} \delta_{\nu}^{Z}$. Since cylindrical symmetry guarantees the independence of the field $\bar{\psi}$ on the $Z$ coordinate, we see that our gauge for the linearized theory satisfies the de Donder conditions, in agreement with our comments above.

Actually, the relation between the linearization of the AP gauge, on the one hand, and the de Donder gauge for the linearized ER model, on the other, is even tighter. In general relativity, the de Donder conditions select not just one, but a family of gauges, leaving a remaining freedom in the choice of coordinates [23]. For ER waves in linearized gravity, however, it is possible to see that the de Donder gauge is unique 
if one fixes the location of the symmetry axis at $R=0$ and imposes there (the linearized counterpart of) the regularity conditions (2.4) and (2.6).

Let us prove this assertion. From Eq. (4.1) and the comments above it, we see that, at linear order in the fields, the regularity conditions (2.4) amount to

$$
\bar{\rho}(R=0)=\bar{\rho}^{\prime}(R=0)=\bar{\gamma}(R=0)=\bar{N}^{R}(R=0)=0 .
$$

Besides, the stability under diffeomorphisms of the regularity conditions demands the restriction (2.6), which translates into

$$
\left(\bar{N}^{R}\right)^{\prime}(R=0)=0 .
$$

On the other hand, the de Donder conditions $\bar{h}_{\mu \nu}{ }^{\nu}=0$ for the cylindrically symmetric metric (3.1) are equivalent to the equations

$$
\begin{array}{r}
\left(\bar{N}^{R}\right)^{\prime}+\dot{\bar{N}}-\frac{\dot{\bar{\gamma}}}{2}-\frac{8 G \dot{\bar{\rho}}}{R}=0, \\
\dot{\bar{N}}^{R}+\bar{N}^{\prime}-\frac{\bar{\gamma}^{\prime}}{2}-\frac{\bar{\gamma}}{R}+\frac{8 G \bar{\rho}^{\prime}}{R}+\frac{8 G \bar{\rho}}{R^{2}}=0 .
\end{array}
$$

The linearized Hamiltonian constraint in Eq. (4.4) and the regularity conditions (5.2) imply that

$$
\bar{\gamma}=16 G \bar{\rho}^{\prime} .
$$

Recalling the Hamilton equations (4.3), the radial constraint of the linearized theory is then straightforwardly satisfied. In addition, differentiating Eqs. (5.4) and (5.5) with respect to $R$ and $t$, respectively, subtracting the results, and substituting relation (5.6), we arrive at

$$
\left(\bar{N}^{R}\right)^{\prime \prime}-\ddot{\bar{N}}^{R}=0 .
$$

So $\bar{N}^{R}$ can be expanded in terms of "plane" waves $e^{i k(t+R)}$ and $e^{i k(t-R)}$, with $k \in \mathbb{R}$. But the only superposition of these waves that satisfies the conditions $\bar{N}^{R}=\left(\bar{N}^{R}\right)^{\prime}=0$ at $R=0$ for all times [see requirements (5.2) and (5.3)] is the zero field. For $\bar{N}^{R}=0$ and $\bar{\gamma}=16 G \bar{\rho}^{\prime}$, the de Donder equations (5.4) and (5.5) reduce simply to

$$
\bar{N}=8 G \bar{\rho}^{\prime}+\frac{8 G \bar{\rho}}{R}+c,
$$

with $c$ being a constant.

We have not used yet the equation of motion for $\bar{\rho}$ in the linearized system. This equation can be easily deduced from the linearized action (4.2), taking into account the Hamilton equations (4.3) and the form of the linearized constraints:

$$
8 G \ddot{\bar{\rho}}-\bar{N}^{\prime}-\dot{\bar{N}}^{R}=0
$$

It is possible to see that, given relation (5.6), the only other independent equation of motion left in the system is that for the field $\bar{\psi}$, which percolates to the reduced model. With $\bar{N}^{R}=0$ and formula (5.7), the above equation for $\bar{\rho}$ translates into

$$
\ddot{\bar{\rho}}=\bar{\rho}^{\prime \prime}+\frac{\bar{\rho}^{\prime}}{R}-\frac{\bar{\rho}}{R^{2}},
$$

which admits solutions of the form $e^{i k t} J_{1}(R k)$ and $e^{i k t} Y_{1}(R k), k \in \mathrm{R}$, in terms of first-order Bessel functions [26]. Again, the only solution of this type allowed by our regularity conditions at the axis, which demand that $\bar{\rho}$ and $\bar{\rho}^{\prime}$ vanish at $R=0$, is the zero function. Since $\bar{\rho}$ vanishes, Eq. (5.7) implies then that $\bar{N}$ must be constant. This constant can be set equal to zero by requiring a vanishing excess of the lapse with respect to the Minkowski background, either at the symmetry axis or at spatial infinity.

In conclusion, we have shown that the de Donder conditions, together with our regularity requirements at $R=0$, completely determine the gauge choice for ER waves in linearized gravity. The gauge fixing is such that all metric fields vanish except $\bar{\psi}$. The resulting reduced metric is just that found in Sec. IV for the linearized model, namely the metric obtained with the linearization of the gauge conditions selected by Ashtekar and Pierri. As a consequence, the gauge chosen in the AP formulation is a valid generalization of the de Donder gauge from the linearized to the full ER model.

\section{ANNIHILATION AND CREATION VARIABLES}

In order to discuss the connection between the Fock space employed in the AP quantization of the ER model and that which would arise in its perturbative quantization, we will analyze in this section the relation between the annihilation and creation variables that are associated with each of these two approaches.

As we have commented, the basic metric fields in standard perturbative treatments of gravity, $\bar{h}_{\mu \nu}$, are linear in the excess of the metric around Minkowski. In the linearized theory, one chooses a gauge that simplifies the corresponding equations of motion, for instance a de Donder gauge. When the linearized description is modified by allowing the presence of gravitational interactions, this gauge can be corrected with terms that are of higher order in the fields, both to ensure that the gauge continues to be well posed and to facilitate the analysis of the system. In Sec. V we showed that the gauge choice made by Ashtekar and Pierri is precisely a modified de Donder gauge of this type. We will therefore select it as a valid gauge to compare the results of the AP and the perturbative approaches to the quantization of the ER model.

From this perspective, the relation between the two approaches is based just on a field redefinition, namely the transformation $\psi=\ln (1+\bar{\psi})$ mapping the field $\psi$ of the AP formulation to the field $\bar{\psi}$, which describes the difference with respect to the Minkowski background of the diagonal $Z$ 
component of the metric. In the reduced ER model, this field redefinition becomes a canonical transformation when completed with the momentum change (3.8). The relation that we are interested in is that between the annihilation and creation variables associated with each of the canonical pairs $\left(\psi, p_{\psi}\right)$ and $(\bar{\psi}, \bar{P} \bar{\psi})$.

Although the fields considered are time dependent, we will see below that, for our purposes, it will suffice to study their relation on the initial time surface. If we wanted to analyze the dynamics using the standard techniques of perturbative gravity, we would be forced to consider the evolution in the auxiliary time $T$ of the ER model, rather than in the physical time $t$, at least in a first step. The reason is that the Lagrangian of the reduced model is local only in the former case. For the physical time, the action can be regarded as the sum of a local, a bilocal, and in general multilocal terms of all orders. Once we had dealt with the gravitational interactions in the auxiliary time by perturbative methods, we could change the dynamical description to the physical time in a second step, taking into account the backreaction produced by the presence of gravitational waves in the form of a deficit angle at spatial infinity, accompanied by a modification of the norm of the asymptotic time-like Killing vector.

This philosophy is in fact similar to that adopted in the discussion of cylindrical gravitational waves with general polarization as a sigma model $[28,29]$ (proposed as an alternative approach to other quantization schemes [30]). In this case, the gravitational action has also been made local with the choice of an auxiliary time whose norm at spatial infinity, though constant in the evolution, differs from the unity. The change to the physical time is energy dependent, and leads to a multi-local action.

It is instructive to see the expression of the reduced action of the ER model corresponding to the auxiliary time $T$ in terms of the two types of fields employed to describe the system, namely $\psi$ and $\bar{\psi}$. Remembering that, in the time $T$, the dynamical generator is the Hamiltonian $H_{0}=\gamma_{\infty} /\left(8 G_{3}\right)$ and using relation (2.8), one can check that the associated reduced Lagrangian is

$$
\begin{aligned}
L_{0} & =\frac{1}{8 G_{3}} \int_{0}^{\infty} d R \frac{R}{2}\left[-\left(\psi^{\prime}\right)^{2}+\left(\partial_{T} \psi\right)^{2}\right] \\
& =\frac{1}{8 G_{3}} \int_{0}^{\infty} d R \frac{R}{2(1+\bar{\psi})^{2}}\left[-\left(\bar{\psi}^{\prime}\right)^{2}+\left(\partial_{T} \bar{\psi}\right)^{2}\right] .
\end{aligned}
$$

Thus, while the AP formulation consists of a free-field parametrization of the reduced system, described by $\psi$, the other parametrization, natural from the viewpoint of a perturbative approach, leads to a field $\bar{\psi}$ with self-interactions of all orders, namely

$$
L_{0}=\sum_{n=0}^{\infty}(-1)^{n} \frac{(n+1)}{8 G_{3}} \int_{0}^{\infty} d R \frac{R}{2} \bar{\psi}^{n}\left[-\left(\bar{\psi}^{\prime}\right)^{2}+\left(\partial_{T} \bar{\psi}\right)^{2}\right] .
$$

In this sense, one can interpret the AP formulation as a freefield realization of the ER model.
Regardless of the time parameter selected to describe the evolution of the ER model (the auxiliary or the physical one), the initial time section of the system coincides in both cases, since the times differ only by a positive normalization factor. When quantizing the system, a Fock space is assigned to this initial section. This space being the same for the two natural choices of Hamiltonian, both lead to unitarily equivalent Fock quantizations [14]. Therefore, in order to study the relation between the Fock spaces of the AP and the perturbative approaches, we can restrict all considerations just to the initial time surface $T=t=0$, as we anticipated. Thus, from now on, by $\psi(R), p_{\psi}(R), \bar{\psi}(R)$, and $\bar{P}_{\bar{\psi}}(R)$ we will understand the initial values of these fields.

In the AP description, one introduces annihilation and creation variables, $A(k)$ and $A^{\dagger}(k)\left(k \in \mathbb{R}^{+}\right)$, corresponding to the expansion of the cylindrically symmetric field $\psi$ in terms of zeroth-order Bessel functions. Employing the form of $\psi$ on classical solutions given in Sec. II, the Hamiltonian equation $\partial_{T} \psi=8 G p_{\psi} / R$ and the identity

$$
\int_{0}^{\infty} d R R k J_{0}(R k) J_{0}(R \widetilde{k})=\delta(k, \widetilde{k}),
$$

one can check that

$$
A(k)=\int_{0}^{\infty} d R \frac{J_{0}(R k)}{2 \sqrt{4 G}}\left[R k \psi(R)+i 8 G p_{\psi}(R)\right] .
$$

The complex conjugate of this relation provides $A^{\dagger}(k)$.

In fact, recalling that $\psi$ and $p_{\psi}$ are a canonical pair of cylindrically symmetric fields, it is not difficult to see just from Eq. (6.2) that the only non-vanishing Poisson brackets of $A(k)$ and $A^{\dagger}(k)$ are really

$$
\left\{A(k), A^{\dagger}(\widetilde{k})\right\}=-i \delta(k, \widetilde{k}) .
$$

Therefore, without appealing to the explicit form of the classical solutions, we can regard formula (6.2) and its complex conjugate as the definition of a set of annihilation and creation variables corresponding to the field $\psi$. Furthermore, the same arguments apply exactly as well to any other cylindrically symmetric field and its momentum as far as they form a true canonical pair. For instance, we can adopt the point of view of the perturbative approach and consider the pair $\left(\bar{\psi}, \bar{P}_{\bar{\psi}}\right)$ as the fundamental canonical fields. Associated with them, we then introduce the following type of annihilation variables:

$$
a(k)=\int_{0}^{\infty} d R \frac{J_{0}(R k)}{2 \sqrt{4 G}}\left[R k \bar{\psi}(R)+i 8 G \bar{P}_{\bar{\psi}}(R)\right],
$$

with their complex conjugates providing the creation variables $a^{\dagger}(k)$.

A point that is worth remarking is that the above definitions are the natural ones from the perspective of the perturbative approach. In the linearized gravitational theory, $\bar{\psi}$ satisfies the same cylindrical wave equation as $\psi$ does in the full reduced ER model. Thus, the associated expansion of 
$\bar{\psi}(R, T)$ in terms of complex exponentials of $T$ and Bessel functions of $R$ leads precisely to the above annihilation and creation variables in linearized gravity. In other words, the underlying mode decomposition of the fields $\psi$ and $\bar{\psi}$ is the same at first perturbative order. Actually, since $\left(\bar{\psi}, \bar{P}_{\bar{\psi}}\right)$ and $\left(\psi, p_{\psi}\right)$ coincide at linear order, the series expansion of $A(k)$ in powers of the over-barred fields leads from Eq. (6.2) to definition (6.3) as the leading term in a perturbative expansion (the same line of reasoning applies to the complex conjugate formulas for $A^{\dagger}$ and $a^{\dagger}$ ).

Employing Eq. (6.1), the introduced definitions of creation and annihilation variables can be inverted to obtain the initial values of the fields:

$$
\begin{aligned}
\psi(R) & =\sqrt{4 G} \int_{0}^{\infty} d k J_{0}(R k)\left[A(k)+A^{\dagger}(k)\right], \\
p_{\psi}(R) & =\frac{i R}{\sqrt{16 G}} \int_{0}^{\infty} d k k J_{0}(R k)\left[-A(k)+A^{\dagger}(k)\right],
\end{aligned}
$$

and similar expressions for $\bar{\psi}$ and $\bar{P}_{\bar{\psi}}$. Note again that, in arriving at these formulas, we have not used the explicit form of the classical solutions. They are simply Bessel expansions of the initial fields. The dynamics is encoded in the obviated evolution of the annihilation and creation variables, which have been restricted in our analysis to the initial time surface.

By combining Eqs. (6.3), (3.2), (3.8), and (6.4), it is now straightforward to deduce the highly non-linear relation that exists between the particle-like variables of the AP and the perturbative approaches:

$$
\begin{aligned}
a(k)= & \int_{0}^{\infty} d R \frac{J_{0}(R k)}{2 \sqrt{4 G}}\left[R k \bar{\psi}\left(R \mid A, A^{\dagger}\right)\right. \\
& \left.+i 8 G \bar{P}_{\bar{\psi}}\left(R \mid A, A^{\dagger}\right)\right], \\
\bar{\psi}\left(R \mid A, A^{\dagger}\right)= & e^{\psi\left(R \mid A, A^{\dagger}\right)}-1 \\
:= & \exp \left\{\sqrt{4 G} \int_{0}^{\infty} d \widetilde{k} J_{0}(R \widetilde{k})\left[A(\widetilde{k})+A^{\dagger}(\widetilde{k})\right]\right\} \\
& -1, \\
i 8 G \bar{P}_{\bar{\psi}}(R \mid A)= & i 8 G p_{\psi}\left(R \mid A, A^{\dagger}\right) e^{-\psi\left(R \mid A, A^{\dagger}\right)} \\
:= & \sqrt{4 G} R \int_{0}^{\infty} d \widetilde{k} \widetilde{k} J_{0}(R \widetilde{k})\left[A(\widetilde{k})-A^{\dagger}(\widetilde{k})\right] \\
& \times \exp \left\{-\sqrt{4 G} \int_{0}^{\infty} d \breve{k} J_{0}(R \breve{k})[A(\breve{k})\right. \\
& \left.\left.+A^{\dagger}(\breve{k})\right]\right\},
\end{aligned}
$$

while $a^{\dagger}(k)$ is the complex conjugate of $a(k)$. Note that in fact these definitions implement the reality condition
$\bar{\psi}\left(R \mid A, A^{\dagger}\right)>-1$ (ensuring that the induced metric is positive definite) if $A(k)$ and $A^{\dagger}(k)$ are complex conjugate to each other, because then $\psi\left(R \mid A, A^{\dagger}\right)$ is real everywhere. On the other hand, the inverse transformation between the two sets of particle-like variables can be obtained by substituting the relations $\psi=\ln (1+\bar{\psi})$ and $p_{\psi}=\bar{P}_{\bar{\psi}}(1+\bar{\psi})$ in formula (6.2) and expressing the initial values of $\bar{\psi}$ and $\bar{P}_{\bar{\psi}}$ in terms of $a(k)$ and $a^{\dagger}(k)$ [using the analogue of Eq. (6.4)]. Finally we point out that, in formula (6.5), each of the AP annihilation and creation variables appears multiplied by a factor of $\sqrt{G}$. As a result, one can understand the expansion of $a(k)$ and $a^{\dagger}(k)$ in powers of such variables as equivalent to a perturbative expansion in powers of $\sqrt{G}$.

\section{REGULARIZED OPERATORS}

Once one has established the relation between the sets of annihilation and creation variables associated with the perturbative analysis of the system and with the AP formulation, a natural way to elucidate whether the two schemes lead to equivalent Fock quantizations is the following. One can first try to implement the variables $a(k)$ and $a^{\dagger}(k)$ (associated with the perturbative approach) as annihilation and creation operators acting on the Fock space of the AP quantization. The perturbative vacuum would then be the (unique) state annihilated by all the operators $\hat{a}(k)$. If this state is physical, i.e., if its norm is finite, it determines the Fock space of the perturbative approach. The two considered Fock quantizations would then be unitarily equivalent, the equivalence being given by the map from the perturbative to the AP vacuum. On the contrary, the Fock quantizations would be inequivalent if the perturbative vacuum is not normalizable.

Remembering relation (6.5) (and its complex conjugate), we might naively attempt to promote $a(k)$ and $a^{\dagger}(k)$ to operators in the AP quantization by replacing the variables $A(k)$ and $A^{\dagger}(k)$ with their operator counterpart. However, this procedure fails because, in the quantum version of expression (6.5), the fields $\hat{\psi}(R)$ and $\hat{p}_{\psi}(R)$ that one obtains are not proper operators, but operator-valued distributions [10]. In particular, the exponential of $\pm \hat{\psi}(R)$ is not rigorously defined.

These problems can be overcome by regularizing the fields. We will only consider regularizations that consist of a cutoff $k_{c}$ in wave numbers (or, equivalently, in momentum space), so that they can be described by a regulator $g(k)$ of the form (2.12). Recall that in this case $g^{2}(k)=g(k)$. The corresponding regularized quantum field $\hat{\psi}(R \mid g)$ is given in Eq. (2.11) and is self-adjoint for every $k_{c}<\infty$. The spectral theorem allows us then to define the exponential $e^{ \pm \hat{\psi}(R \mid g)}$ as a positive operator [10]. Employing the Campbell-Baker Hausdorff formula $e^{\hat{b}+\hat{c}}=e^{-[\hat{b}, \hat{c}] / 2} e^{\hat{b}} e^{\hat{c}}$, which is valid for operators $\hat{b}$ and $\hat{c}$ whose commutator is a $c$ number, one can see

$$
e^{ \pm \hat{\psi}(R \mid g)}=e^{2 G|| J_{0}\left(R^{*}\right) g \|}: e^{ \pm \hat{\psi}(R \mid g)}:
$$

where the colon denotes normal ordering and 


$$
|| J_{0}\left(R^{*}\right) g \|=\int_{0}^{\infty} d k\left|J_{0}(R k) g(k)\right|^{2}
$$

For the regularization of the product $i 8 G p_{\psi} e^{-\psi}$ that appears in Eq. (6.5), we choose the ordering

$$
\begin{aligned}
i 8 G( & \left.\hat{p}_{\psi}(R \mid g) e^{-\hat{\psi}(R \mid g)}\right)_{\mathcal{N}} \\
:= & -\sqrt{4 G} R \int_{0}^{k_{c}} d k k J_{0}(R k)\left[\hat{A}^{\dagger}(k) e^{-\hat{\psi}(R \mid g)}\right. \\
& \left.-e^{-\hat{\psi}(R \mid g)} \hat{A}(k)\right] .
\end{aligned}
$$

Finally, we define the following annihilation- and creationlike operators corresponding to $a(k)$ and $a^{\dagger}(k)$ for $k \leqslant k_{c}$ :

$$
\begin{aligned}
\hat{a}(k \mid g):= & g(k) \int_{0}^{\infty} d R \frac{J_{0}(R k)}{2 \sqrt{4 G}}\left[R k e^{\hat{\psi}(R \mid g)}-R_{g}(G) k\right. \\
& \left.+i 8 G\left(\hat{p}_{\psi}(R \mid g) e^{-\hat{\psi}(R \mid g)}\right)_{\mathcal{N}}\right], \\
\hat{a}^{\dagger}(k \mid g):= & g(k) \int_{0}^{\infty} d R \frac{J_{0}(R k)}{2 \sqrt{4 G}}\left[R k e^{\hat{\psi}(R \mid g)}-R_{g}(G) k\right. \\
& \left.-i 8 G\left(\hat{p}_{\psi}(R \mid g) e^{-\hat{\psi}(R \mid g)}\right)_{\mathcal{N}}\right] .
\end{aligned}
$$

By construction, these operators are adjoint to each other. In order to account for part of the order ambiguity, we have left the freedom to represent $R$ quantum mechanically by a $c$ number $R_{g}(G)$ that may depend on the cutoff, as well as on the quantum gravitational constant $G$. To recover the semiclassical limit, we impose the condition that $R_{g}$ tend to $R$ in the limit $G \rightarrow 0$. For simplicity, we also assume that $R_{g}(G)$ is analytic in $G$.

The commutators of the operators (7.3) are computed in the Appendix. Of course, they do not depend on the form of the $c$ number $R_{g}(G)$. We will only comment two important properties of these commutators. First, using that $e^{ \pm \hat{\psi}(R \mid g)}$ tends to the identity operator in the limit of vanishing $G$ and remembering the integral expression (6.1), one can check that the only non-vanishing commutators of our operators when $G \rightarrow 0$ are

$$
\lim _{G \rightarrow 0}\left[\hat{a}(k \mid g), \hat{a}^{\dagger}(\widetilde{k} \mid g)\right]=g(k) \delta(k, \widetilde{k}) .
$$

So, in this kind of semiclassical limit, we recover the algebra of a set of annihilation and creation operators in the region of wave numbers to which we are restricting our analysis. Second, one can proceed to remove the cutoff by taking the limit $g(k) \rightarrow 1$ or, equivalently, $k_{c} \rightarrow \infty$. Assuming that this limit can be taken inside the integrals in expression (A1) and using the identity (6.1), a careful calculation shows that

$$
\begin{aligned}
& \lim _{g \rightarrow 1}[\hat{a}(k \mid g), \hat{a}(\widetilde{k} \mid g)]=\lim _{g \rightarrow 1}\left[\hat{a}^{\dagger}(k \mid g), \hat{a}^{\dagger}(\widetilde{k} \mid g)\right]=0, \\
& \lim _{g \rightarrow 1}\left[\hat{a}(k \mid g), \hat{a}^{\dagger}(\widetilde{k} \mid g)\right]=\delta(k, \widetilde{k}) .
\end{aligned}
$$

In this sense, the desired algebra of annihilation and creation operators associated with the perturbative quantization scheme can be regarded as the limit of our algebra of operators when the cutoff is driven to infinity.

Substituting the regulated expressions (2.11) and (7.2) in the definition of the operators $\hat{a}(k \mid g)$ and $\hat{a}^{\dagger}(k \mid g)$, it is not difficult to obtain their expansion in powers of annihilation and creation operators of the AP quantization, $\hat{A}(k)$ and $\hat{A}^{\dagger}(k)$. According to our comments at the end of Sec. VI, this expansion reproduces the power series in $\sqrt{G}$, except for the possible $G$ dependence introduced by the $c$ number $R_{g}(G)$, which (partially) accounts for operator ordering ambiguities. Therefore, one can interpret the series in $\sqrt{G}$ in the sense that each additional power corresponds (in a certain operator ordering) to the creation or annihilation of an extra particle in the AP quantization. Explicitly, the series will have the form

$$
\begin{aligned}
& \hat{a}(k \mid g)=\sum_{n=0}^{\infty}(G)^{n / 2} \hat{a}_{(n)}(k \mid g), \\
& \hat{a}^{\dagger}(k \mid g)=\sum_{n=0}^{\infty}(G)^{n / 2} \hat{a}_{(n)}^{\dagger}(k \mid g),
\end{aligned}
$$

where now the operators $\hat{a}_{(n)}(k \mid g)$ and their adjoints are independent of the quantum gravitational constant $G$.

Remembering that $R_{g}(G)$ is analytic in $G$ and equal to $R$ at $G=0$, and using identity (6.1), it is easy to find the zerothorder contribution to $\hat{a}(k \mid g)$ :

$$
\begin{aligned}
\hat{a}_{(0)}(k \mid g) & =g(k) \int_{0}^{\infty} d R \frac{J_{0}(R k)}{2 \sqrt{4 G}}\left[R k \hat{\psi}(R \mid g)+i 8 G \hat{p}_{\psi}(R \mid g)\right] \\
& =g(k) \hat{A}(k) .
\end{aligned}
$$

Similarly, $\hat{a}_{(0)}^{\dagger}(k \mid g)=g(k) \hat{A}^{\dagger}(k)$. Thus, our definition of annihilation- and creation-like operators for the perturbative approach is such that, in the considered sector of wave numbers $k \leqslant k_{c}$, they coincide with the annihilation and creation operators of the AP formulation at dominant order in $\sqrt{G}$. In other words, at first perturbative order the two types of particles can be identified in the region of momentum space below the cutoff. Note that one can completely determine the particle contain in this perturbative limit by finally proceeding to remove the regulator, i.e. $\hat{A}(k)$ $=\lim _{g \rightarrow 1} \lim _{G \rightarrow 0} \hat{a}(k \mid g)$ for all $k \in \mathbb{R}$.

\section{PERTURBATIVE VACUUM}

We have seen that the algebra of $\hat{a}(k \mid g)$ and $\hat{a}^{\dagger}(k \mid g)$, in the limit of infinite cutoff, has the form of that of a set of annihilation and creation operators. In addition, their values for $G=0$ coincide with the annihilation and creation operators of the AP formulation for wave numbers $k$ smaller than the cutoff, reproducing the whole set of those operators when the regulator disappears. The AP vacuum $|0\rangle$ is hence totally fixed by the condition that it be annihilated by the operators 
$\hat{a}_{(0)}(k \mid g)$ for every value of the cutoff $k_{c}$ or, equivalently, in the limit $k_{c} \rightarrow \infty$. Besides, it is possible to check that $|0\rangle$ is not annihilated by all the operators $\hat{a}(k \mid g)$ for each fixed $k_{c}>0$, and so neither is it when the regulator is removed. Therefore, the AP vacuum differs from that corresponding to the perturbative approach, which we will call $|\overline{0}\rangle$.

Provided that the latter of these vacua belongs to the Fock space of the AP quantization, a way to determine it is the following. First, for each fixed cutoff, we find a state $\left|\overline{0}_{g}\right\rangle$ annihilated by all the operators $\hat{a}(k \mid g)$ and such that coincides with the AP vacuum $|0\rangle$ in the limit $G \rightarrow 0$. If $\left|\overline{0}_{g}\right\rangle$ is a physical state, one can choose it with unit norm. The vacuum $|\overline{0}\rangle$ would then be attained as the limit of this normalized state when the regulator is removed, $k_{c} \rightarrow \infty$.

Note however that our definition (7.3) of $\hat{a}(k \mid g)$ involves only annihilation and creation operators of the AP formulation with $k \leqslant k_{c}$. As a consequence, one can anticipate the existence of ambiguities in the determination of $\left|\overline{0}_{g}\right\rangle$, owing to a lack of uniqueness in the allowed contributions from the sector of AP particles with wave number greater than the cutoff. Nonetheless, this ambiguity can be eliminated by demanding that the regularized perturbative vacuum $\left|\overline{0}_{g}\right\rangle$ has no projection in that sector. This is a natural condition if we interpret the regularization as the removal of all interactions and particles with energies above the cutoff. In particular, it is consistent with the requirement that the limit of $\left|\overline{0}_{g}\right\rangle$ when $G$ tends to zero be the AP vacuum $|0\rangle$, because this is the only physical state which does not contain particles with $k>k_{c}$ and is annihilated by all the operators $\hat{a}_{(0)}(k \mid g)$ [which are equal to $\hat{A}(k)$ below the cutoff and vanish otherwise].

The computation of $\left|\overline{0}_{g}\right\rangle$ can be carried out perturbatively in terms of the quantum gravitational constant $G$. In order to do it, one employs the series (7.4) for the annihilation-like operators $\hat{a}(k \mid g)$ and expands the regularized vacuum as a formal power series of $\sqrt{G}$ as well:

$$
\left|\overline{0}_{g}\right\rangle=|0\rangle+\sum_{n=1}^{\infty}(G)^{n / 2}\left|\Phi_{n, g}\right\rangle
$$

Here, we have made explicit that the dominant contribution must be the vacuum $|0\rangle$, and the $\left|\Phi_{n, g}\right\rangle$ 's designate linear superpositions of states with a finite, non-zero number of AP particles whose wave number is bounded by the cutoff, $k$ $\leqslant k_{c}$. The above formula can equivalently be regarded as providing the AP vacuum $|0\rangle$ as a formal series in $\sqrt{G}$ in terms of the regularized perturbative vacuum, $|0\rangle=\left|\overline{0}_{g}\right\rangle$ $-\Sigma(G)^{n / 2}\left|\Phi_{n, g}\right\rangle$.

By an iterative process, one can deduce the form of all the states $\left|\Phi_{n, g}\right\rangle$. Namely, once $\left|\Phi_{n, g}\right\rangle$ is known for every $n<m$, one can determine $\left|\Phi_{m, g}\right\rangle$ from the condition that $\hat{a}(k \mid g)\left|\overline{0}_{g}\right\rangle$ vanish for all $k$ at order $G^{m / 2}$. Let us consider the case $m=1$ :

$$
\hat{a}_{(0)}(k \mid g)\left|\Phi_{1, g}\right\rangle+\hat{a}_{(1)}(k \mid g)|0\rangle=0 .
$$

From Eq. (7.3) and the analyticity of $R_{g}(G)$ in $G$, one can see that

$$
\begin{aligned}
\hat{a}_{(1)}(k \mid g)|0\rangle= & \frac{g(k)}{2}\left[\int_{0}^{\infty} d R J_{0}(R k) k E_{g}(R)|0\rangle\right. \\
& +\int_{0}^{k_{c}} d k_{1} \int_{0}^{k_{c}} d k_{2}\left(k_{1}+k_{2}+k\right) \\
& \left.\times F\left(k_{1}, k_{2}, k\right) \hat{A}^{\dagger}\left(k_{1}\right) \hat{A}^{\dagger}\left(k_{2}\right)|0\rangle\right],
\end{aligned}
$$

where we have defined the functions

$$
\begin{aligned}
E_{g}(R) & :=\left\|J_{0}\left(R^{*}\right) g\right\| R-\frac{1}{2} \partial_{G} R_{g}(G=0), \\
F\left(k_{1}, k_{2}, k_{3}\right) & :=\int_{0}^{\infty} d R R J_{0}\left(R k_{1}\right) J_{0}\left(R k_{2}\right) J_{0}\left(R k_{3}\right),
\end{aligned}
$$

and we have interchanged the order of integration in $R$ and in $\left(k_{1}, k_{2}\right)$. The first term in $E_{g}(R)$ arises from the derivative with respect to $G$ of the factor $e^{2 G|| J_{0}\left(R^{*}\right) g \|}$, which appears in the operator $e^{\hat{\psi}(R \mid g)}$ when expressed in normal ordering [see Eq. (7.1)]. On the other hand, notice that the function $F\left(k_{1}, k_{2}, k_{3}\right)$ is symmetric in all its arguments.

Condition (8.2) implies then that

$$
\left|\Phi_{1, g}\right\rangle=-\int_{0}^{k_{c}} d k_{1} \frac{k_{1}}{2} \int_{0}^{\infty} d R J_{0}\left(R k_{1}\right) E_{g}(R) \hat{A}^{\dagger}\left(k_{1}\right)|0\rangle-\left|\Upsilon_{g}\right\rangle,
$$

with

$$
\begin{aligned}
\left|\Upsilon_{g}\right\rangle:= & \int_{0}^{k_{c}} d k_{1} \int_{0}^{k_{c}} d k_{2} \int_{0}^{k_{c}} d k_{3} \frac{\left(k_{1}+k_{2}+k_{3}\right)}{6} \\
& \times F\left(k_{1}, k_{2}, k_{3}\right) \hat{A}^{\dagger}\left(k_{1}\right) \hat{A}^{\dagger}\left(k_{2}\right) \hat{A}^{\dagger}\left(k_{3}\right)|0\rangle .
\end{aligned}
$$

Any possible contribution to $\left|\Phi_{1, g}\right\rangle$ proportional to the vacuum has been obviated, because it is not necessary to satisfy condition (8.2). Moreover, such a contribution can always be absorbed in the dominant term of the series expansion (8.1) up to a $G$-dependent, global numeric factor in $\left|\overline{0}_{g}\right\rangle$ that only changes the norm of this state.

Similar arguments can be applied to fix the next correction to the AP vacuum, $\left|\Phi_{2, g}\right\rangle$, as a superposition of states with a finite but non-zero number of particles belonging to the sector $k \leqslant k_{c}$, using the condition

$$
\hat{a}_{(0)}(k \mid g)\left|\Phi_{2, g}\right\rangle+\hat{a}_{(1)}(k \mid g)\left|\Phi_{1, g}\right\rangle+\hat{a}_{(2)}(k \mid g)|0\rangle=0 .
$$

Likewise, $\left|\Phi_{m, g}\right\rangle$ can be fixed from the corresponding condition at order $G^{m / 2}$ once $\left\{\left|\Phi_{n, g}\right\rangle ; n<m\right\}$ have been determined.

Employing that $\left|\Phi_{1, g}\right\rangle$ is the sum of a one-particle state and the three-particle state $\left|Y_{g}\right\rangle$, which are orthogonal to each other and to the AP vacuum, and the fact that $\left|\Phi_{2, g}\right\rangle$ is a linear combination of states with non-zero particles, so that 
$\left\langle 0 \mid \Phi_{2, g}\right\rangle=0$, we conclude that, up to corrections of order $G^{n / 2}$ with $n \geqslant 3$, the norm of $\left|\overline{0}_{g}\right\rangle$ satisfies

$$
\left\langle\overline{0}_{g} \mid \overline{0}_{g}\right\rangle \geqslant\langle 0 \mid 0\rangle+G\left\langle\Upsilon_{g} \mid \Upsilon_{g}\right\rangle=1+G\left\langle\Upsilon_{g} \mid \Upsilon_{g}\right\rangle
$$

We will now show that the norm of $\left|Y_{g}\right\rangle$ is infinite regardless of the value of the cutoff, and therefore $\left|\overline{0}_{g}\right\rangle$ is not a physical state (at least as a power series in $\sqrt{G}$ ). Thus, one cannot reach in this way a normalized perturbative vacuum in the limit $k_{c} \rightarrow \infty$. This strongly indicates that the perturbative vacuum is not included in the Fock space of the AP formulation, implying that the two discussed approaches to the quantization of the ER waves are unitarily inequivalent. In any case, the non-normalizability of $\left|Y_{g}\right\rangle$ means that the perturbative vacuum is not analytic in $\sqrt{G}$, invalidating the perturbative calculation presented above.

The norm of $\left|Y_{g}\right\rangle$ is given by

$$
\begin{aligned}
\left\langle Y_{g} \mid Y_{g}\right\rangle= & \int_{0}^{k_{c}} d k_{1} \int_{0}^{k_{c}} d k_{2} \int_{0}^{k_{c}} d k_{3} \frac{\left(k_{1}+k_{2}+k_{3}\right)^{2}}{6} \\
& \times F^{2}\left(k_{1}, k_{2}, k_{3}\right) .
\end{aligned}
$$

So, in order to obtain it, we will first calculate the integral (8.3), which provides the function $F\left(k_{1}, k_{2}, k_{3}\right)$. This integral can be computed explicitly, e.g. using the formulas of Ref. [31]. The result is

$$
\begin{aligned}
F\left(k_{1}, k_{2}, k_{3}\right)= & \Theta\left(k_{1}+k_{2}-k_{3}\right) \Theta\left(k_{3}-\left|k_{1}-k_{2}\right|\right) \\
& \times \frac{2}{\pi \sqrt{4 k_{1}^{2} k_{2}^{2}-\left(k_{1}^{2}+k_{2}^{2}-k_{3}^{2}\right)^{2}}},
\end{aligned}
$$

where $\Theta(k)$ is the Heaviside step function, equal to the unity if $k$ is positive and vanishing otherwise. One therefore arrives at

$$
\begin{aligned}
\left\langle Y_{g} \mid Y_{g}\right\rangle & \\
= & \int_{0}^{k_{c}} d k_{1} \int_{0}^{k_{c}} d k_{2} \int_{\left|k_{1}-k_{2}\right|}^{\min \left\{k_{c}, k_{1}+k_{2}\right\}} d k_{3} \frac{\left(k_{1}+k_{2}+k_{3}\right)}{6 \pi^{2}} \\
& \times \frac{1}{\left(k_{1}+k_{2}-k_{3}\right)\left(k_{3}-\left|k_{1}-k_{2}\right|\right)\left(k_{3}+\left|k_{1}-k_{2}\right|\right)} .
\end{aligned}
$$

Here, $\min \{a, b\}$ denotes the minimum of the numbers $a$ and b.

Note that the integrand in the above expression is positive in the integration region, and that the last integral has a simple pole at the boundary $k_{3}=\left|k_{1}-k_{2}\right|$ of the integration interval for $k_{3}$. As a consequence, the integral that determines the norm of $\left|Y_{g}\right\rangle$ diverges for all positive values of the cutoff $k_{c}$. In other words, regardless of the cutoff, $\left|Y_{g}\right\rangle$ is not a physical state. We thus conclude that the vacuum of the perturbative approach is not accessible as a power series in $\sqrt{G}$ in the Fock space of the AP quantization.

It is worth emphasizing that the divergence of the norm of $\left|Y_{g}\right\rangle$ does not arise as a result of taking the limit in which the cutoff is removed because, if that were the case, one could proceed to renormalize the perturbative vacuum. Namely, one could first normalize $\left|\overline{0}_{g}\right\rangle$, obtaining the unit norm state $\left|\widetilde{0}_{g}\right\rangle=\left|\overline{0}_{g}\right\rangle\left[\left\langle\overline{0}_{g} \mid \overline{0}_{g}\right\rangle\right]^{-1 / 2}$, and only then consider the limit $k_{c} \rightarrow \infty$.

\section{SUMMARY AND CONCLUSIONS}

We have investigated the relation between the notion of particle that arises in the quantum framework developed by Ashtekar and Pierri for the description of the ER waves and that expected in a perturbative approach to the quantization of this system. We have started by introducing a set of metric fields that are specially suitable for the analysis of the model in linearized gravity, since the fields are linear in the excess of the metric with respect to the Minkowski background. Using this new field parametrization of the ER spacetimes, we have discussed their quantization in the linearized theory of gravity. We have shown that the linearization of the gauge fixing conditions adopted by Ashtekar and Pierri provides the only gauge choice of the de Donder type which respects the regularity conditions imposed on the metric at the axis of rotational symmetry, located at a fixed location (namely, the origin of the radial coordinate $R$ ). This result allows one to interpret the gauge selected by Ashtekar and Pierri as a wellposed generalization to the ER model of the de Donder gauge compatible with the regularity at the axis.

From this perspective, the perturbative description of the system can be made to rest on a field $\bar{\psi}$, which parametrizes the metric in the AP gauge and is linear in the excess around Minkowski spacetime. By contrast, the parametrization chosen in the AP formulation is based on a field $\psi$ that is highly non-linear in the metric excess, but straightforwardly incorporates the reality conditions on the metric and, more importantly, is subject to a linear (reduced) dynamics. The correspondence between the two fields can be completed into a canonical transformation on the phase space of the reduced ER model. This canonical transformation provides the key relation for discussing the correspondence between the particle-like variables of the two considered descriptions.

Given a field and its canonical momentum, both possessing rotational symmetry and being regular at the symmetry axis, it is possible to expand their initial values in terms of zeroth-order Bessel functions. Using this property, one can associate with the canonical pair of axisymmetric fields on a constant time section a set of annihilation and creation variables with positive wave numbers. This possibility is at hand both for the field $\psi$ and its momentum in the AP formulation and for the field $\bar{\psi}$ and its momentum in the perturbatively inspired description that is linear in the metric excess. The transformation between both pairs of fields provides the relation between the corresponding sets of annihilation and creation variables. Furthermore, since the linearization of both parametrizations is the same, the introduced particlelike variables coincide at linear order.

What one gets in this way is the expression, e.g., of the particle-like variables associated with the perturbative analysis as highly non-linear functionals of the corresponding set 
of AP variables. This expression can be expanded as a power series in the latter set, each new particle-like variable being accompanied by a factor of $\sqrt{G}$. Thus, one can regard the square root of the quantum gravitational constant as the interaction constant of the model and the expansion in the number of created and annihilated particles as a perturbative expansion in that constant. As we have said, the expansion is such that the annihilation and creation variables of the AP formulation are taken as the leading contribution for small $\sqrt{G}$; i.e., the mode decomposition is made to coincide with that of the AP description at dominant perturbative order.

We have next proceeded to consider the quantum version of the relation between the variables of annihilation and creation type for the two formulations. We have employed as starting point the Fock space of the AP quantization, since it provides a mathematically well-posed framework where the quantum issues can be discussed with rigor. The first problem that has been necessary to overcome, in order to promote to meaningful operators the particle-like variables of the perturbative scheme, is to regularize the quantum fields. This has been done by introducing a cutoff in the model and suppressing all particle interactions with energies (or equivalently wave numbers) above it. The resulting operators have been proved to reproduce a formal algebra of annihilation and creation operators in the limit in which the cutoff is removed. In addition, the representation chosen is such that, for vanishing quantum gravitational constant $G$, one exactly recovers the annihilation and creation operators of the AP quantization in the sector of particles with wave numbers below the cutoff.

Using these regularized operators, one can investigate whether the perturbative vacuum can be represented as a physical state in the Fock space of the AP quantization. If this were the case, the two considered quantum theories (namely, those based on the AP and on the perturbative vacuum) would be unitarily equivalent. In more detail, we wanted to elucidate whether there exists a physical state in the Fock space of the AP formalism that can play the role of vacuum in the perturbative approach and be reached in the limit of infinite cutoff as a perturbative power series in the coupling constant $\sqrt{G}$, with interacting-free term (i.e., the contribution at $G=0$ ) given by the original AP vacuum.

The other terms in this (regularized) series for the perturbative vacuum can be determined, for each fixed value of the cutoff, by imposing the condition that they consist of linear superpositions of states with a finite but non-zero number of AP particles, and that they be annihilated by all the regularized annihilation operators. In particular, we have studied in detail the first perturbative correction, proportional to $\sqrt{G}$. It is formed by a three-particle and a one-particle state. More importantly, we have proved that the norm of the three- particle state is infinite regardless of the value of the cutoff. Therefore, no normalizable perturbative vacuum is accessible from the AP vacuum as a power series in $\sqrt{G}$.

This result is a clear indication of the inequivalence of the Fock quantizations associated with the AP and the perturbative approaches. Furthermore, the fact that the perturbative vacuum cannot be realized as a physical state analytic in $\sqrt{G}$ prevents one from applying standard perturbative calculations based on the number of AP particles involved in the interaction, so that a naive perturbative treatment of the system is bound to fail.

As we have commented, the Fock space that we have considered describes the reduced degrees of freedom of the system in a section of constant initial time. In this space one can introduce a quantum dynamics, which provides the evolution of these degrees of freedom as time progresses. In the case of the ER waves, this notion of reduced dynamics can be linked to two types of quantum Hamiltonian, a local one that describes the evolution in an auxiliary time, conformally flat in two dimensions together with the radial coordinate, and a non-local one that corresponds to a physical time, normalized to the unity at spatial infinity. The analysis of the system with the first Hamiltonian is trivial in the AP quantization, in the sense that it leads to a free-field realization. The quantum evolution in the physical time, on the other hand, is much more involved. It can also be studied by a perturbative approach, but this time the perturbative order corresponds to the degree of non-locality. An $n$-point contribution to the Hamiltonian will be proportional to the $(n-1)$ th power of the gravitational constant $G$.

The fact that the same constant $G$ plays the role of perturbative parameter in both types of analyses (one linked to the number of AP particles involved in the local interaction and the other to the degree of non-locality in the dynamics) may lead to some confusion. This is in part due to the fact that the system possesses only a fundamental constant, so that it will show up in any natural expansion. Anyway, a full perturbative analysis can always be made in two steps, first discussing the quantum system at a fixed instant of time, as we have made here, and then taking into account the nonlocality introduced by the change from the auxiliary to the physical time. This second type of perturbative issues will be considered elsewhere [32].

\section{ACKNOWLEDGMENT}

The authors are greatly thankful to M. Varadarajan for suggestions and enlightening conversations. They are also grateful to A. Ashtekar and A. Corichi for valuable questions and discussions. This work was supported by the Spanish MCYT projects BFM2002-04031-C02 and BFM2001-0213.

\section{APPENDIX}

In this appendix we compute the commutators of the operators $\hat{a}(k \mid g)$ and $\hat{a}^{\dagger}(k \mid g)$ defined in Eq. (7.3). Let us introduce the symbolic notation $\hat{a}^{\ddagger}(k \mid g)$ for both types of operators, with $(-1)^{\ddagger}$ equal to -1 and 1 , respectively, in the annihilation and creation case. Using relation (7.1) and the basic commutators (2.9), one can then see that 


$$
\begin{aligned}
{\left[\hat{a}(k \mid g), \hat{a}^{\ddagger}(\widetilde{k} \mid g)\right]=} & g(k) g(\widetilde{k}) \int_{0}^{\infty} d R \frac{R}{2} J_{0}(R k) \int_{0}^{\infty} d \widetilde{R} \widetilde{R} J_{0}(\widetilde{R} \widetilde{k}) \int_{0}^{k_{c}} d k_{1} k_{1} J_{0}\left(R k_{1}\right) \int_{0}^{k_{c}} d k_{2} J_{0}\left(\widetilde{R} k_{2}\right)\left[(-1)^{\ddagger} \sqrt{4 G} k_{2}\right. \\
& \times\left(\left\{J_{0}\left(R k_{2}\right) \hat{A}^{\dagger}\left(k_{1}\right)-J_{0}\left(\widetilde{R} k_{1}\right) \hat{A} \dagger\left(k_{2}\right)\right\} e^{-\hat{\psi}(R \mid g)} e^{-\hat{\psi}(\widetilde{R} \mid g)}+e^{-\hat{\psi}(R \mid g)} e^{-\hat{\psi}(\widetilde{R} \mid g)}\left\{J_{0}\left(\widetilde{R} k_{1}\right) \hat{A}\left(k_{2}\right)\right.\right. \\
& \left.\left.\left.-J_{0}\left(R k_{2}\right) \hat{A}\left(k_{1}\right)\right\}\right)+\delta\left(k_{1}, k_{2}\right)\left\{\widetilde{k} e^{-\hat{\psi}(R \mid g)} e^{\hat{\psi}(\widetilde{R} \mid g)}+(-1)^{\ddagger} k e^{\hat{\psi}(R \mid g)} e^{-\hat{\psi}(\widetilde{R} \mid g)}\right\}\right] .
\end{aligned}
$$

[1] G. Beck, Z. Phys. 33, 713 (1925).

[2] A. Einstein and N. Rosen, J. Franklin Inst. 223, 43 (1937).

[3] K.S. Thorne, Phys. Rev. 138, B251 (1965); M.A. Melvin, ibid. 139, B225 (1965).

[4] See Appendix B in S. Chandrasekhar, Proc. R. Soc. London A408, 209 (1986).

[5] P. Jordan, J. Ehlers, and W. Kundt, Akad. Wiss. Lit. Mainz Abh. Math. Naturwiss. Kl. 1960, No. 2 (1960); A.S. Kompaneets, Zh. Éksp. Teor. Fiz. 34, 953 (1958) [Sov. Phys. JETP 7, 659 (1958)].

[6] K. Kuchař, Phys. Rev. D 4, 955 (1971).

[7] M. Allen, Class. Quantum Grav. 4, 149 (1987).

[8] A. Ashtekar and M. Pierri, J. Math. Phys. 37, 6250 (1996).

[9] M. Varadarajan, Class. Quantum Grav. 17, 189 (2000).

[10] M.E. Angulo and G.A. Mena Marugán, Int. J. Mod. Phys. D 9 , 669 (2000).

[11] A. Ashtekar, Phys. Rev. Lett. 77, 4864 (1996).

[12] R. Gambini and J. Pullin, Mod. Phys. Lett. A A 12, 2407 (1997)

[13] A.E. Domínguez and M.H. Tiglio, Phys. Rev. D 60, 064001 (1999).

[14] J.F. Barbero G., G.A. Mena Marugán, and E.J.S. Villaseñor, Phys. Rev. D 67, 124006 (2003).

[15] M. Niedermaier, Phys. Lett. B 498, 83 (2001).

[16] I. Kouletsis, P. Hájíček, and J. Bičák, Phys. Rev. D 68, 104013 (2003).

[17] J.D. Romano and C.G. Torre, Phys. Rev. D 53, 5634 (1996).

[18] A. Ashtekar and M. Varadarajan, Phys. Rev. D 50, 4944 (1994).
[19] M. Varadarajan, Phys. Rev. D 52, 2020 (1995).

[20] A. Ashtekar, J. Bičák, and B.G. Schmidt, Phys. Rev. D 55, 669 (1997); 55, 687 (1997).

[21] G.A. Mena Marugán, Phys. Rev. D 63, 024005 (2001).

[22] N. Manojlović and G.A. Mena Marugán, Class. Quantum Grav. 18, 2065 (2001).

[23] See, e.g., C.W. Misner, K.S. Thorne, and J.A. Wheeler, Gravitation (Freeman, San Francisco, 1973).

[24] Strictly speaking, we then have $(R / G) \in \mathbb{R}^{+},(Z / G) \in \mathbb{R}$, and $(t / G) \in \mathbb{R}$.

[25] We thank M. Varadarajan for remarking this point.

[26] Handbook of Mathematical Functions, Natl. Bur. Stand. Appl. Math. Ser. No. 55, revised 9th ed., edited by M. Abramowitz and I.A. Stegun (U.S. GPO, Washington, D.C., 1970).

[27] M. Reed and B. Simon, Methods of Modern Mathematical Physics II: Fourier Analysis, Self Adjointness (Academic Press, New York, 1975).

[28] M. Niedermaier, J. High Energy Phys. 12, 066 (2002); Nucl. Phys. B673, 131 (2003).

[29] J.F. Barbero G., G.A. Mena Marugán, and E.J.S. Villaseñor, Int. J. Mod. Phys. D (to be published), gr-qc/0402096.

[30] D. Korotkin and H. Samtleben, Phys. Rev. Lett. 80, 14 (1998).

[31] Employ formula 8 of Chap. 6.57 and formula 8.704 in I.S. Grashteyn and I.M. Ryzhik, Table of Integrals, Series and Products, 5th ed. (Academic Press, London, 1994). For the form of the hypergeometric function involved in these expressions one can use formula 15.1.12 of [26].

[32] J.F. Barbero G., G.A. Mena Marugán, and E.J.S. Villaseñor, J. Math. Phys. (to be published), gr-qc/0405075. 